\title{
The neutral hydrogen content of Fornax cluster galaxies
}

\author{
A. Schröder ${ }^{1}$, M. J. Drinkwater ${ }^{2}$, and O.-G. Richter ${ }^{3}$ \\ 1 Observatoire de la Côte d'Azur, BP 4229, 06304 Nice Cedex 4, France \\ 2 School of Physics, University of Melbourne, Victoria 3010, Australia \\ 3 Hamburger Sternwarte, Gojenbergsweg 112, 20119 Hamburg, Germany
}

Received 7 March 2001 / Accepted 5 July 2001

\begin{abstract}
We present a new set of deep H I observations of member galaxies of the Fornax cluster. We detected 35 cluster galaxies in HI. The resulting sample, the most comprehensive to date, is used to investigate the distribution of neutral hydrogen in the cluster galaxies. We compare the $\mathrm{H}$ i content of the detected cluster galaxies with that of field galaxies by measuring H I mass-to-light ratios and the H I deficiency parameter of Solanes et al. (1996). The mean H I mass-to-light ratio of the cluster galaxies is $0.68 \pm 0.15$, significantly lower than for a sample of $\mathrm{H}$ I-selected field galaxies (1.15 \pm 0.10$)$, although not as low as in the Virgo cluster $(0.45 \pm 0.03)$. In addition, the H I content of two cluster galaxies (NGC 1316C and NGC 1326B) appears to have been affected by interactions. The mean $\mathrm{H}$ I deficiency for the cluster is $0.38 \pm 0.09$ (for galaxy types $T=1-6$ ), significantly greater than for the field sample $(0.05 \pm 0.03)$. Both these tests show that Fornax cluster galaxies are H I-deficient compared to field galaxies. The kinematics of the cluster galaxies suggests that the H I deficiency may be caused by ram-pressure stripping of galaxies on orbits that pass close to the cluster core. We also derive the most complete $B$-band Tully-Fisher relation of inclined spiral galaxies in Fornax. A subcluster in the South-West of the main cluster contributes considerably to the scatter. The scatter for galaxies in the main cluster alone is 0.50 mag, which is slightly larger than the intrinsic scatter of 0.4 mag. We use the Tully -Fisher relation to derive a distance modulus of Fornax relative to the Virgo cluster of $-0.38 \pm 0.14$ mag. The galaxies in the subcluster are $(1.0 \pm 0.5) \mathrm{mag}$ brighter than the galaxies of the main cluster, indicating that they are situated in the foreground. With their mean velocity $95 \mathrm{~km} \mathrm{~s}^{-1}$ higher than that of the main cluster we conclude that the subcluster is falling into the main Fornax cluster.
\end{abstract}

Key words. galaxies: clusters: general - galaxies: clusters: individual: Fornax - galaxies: fundamental parameters galaxies: general - radio lines: galaxies

\section{Introduction}

The nearby Fornax cluster has a high central surface density of galaxies (Ferguson 1989b) although it is not a rich cluster in Abell's (1956) sense. It forms an intermediate sample suitable for comparison with richer clusters like Virgo (Huchtmeier \& Richter 1989a; Cayatte et al. 1994) and Hydra I (McMahon et al. 1992), as well as smaller groups and nearby field galaxies (Huchtmeier \& Richter 1988; Marquarding 2000).

In particular, Fornax lies almost opposite in the sky from the well-studied Virgo cluster but at a distance just about equal to it (e.g., Pierce 1989; Bridges et al. 1991; Hamuy et al. 1991; McMillan et al. 1993; see also Table 6.1 in Schröder 1995). A better understanding of the relative distance and the substructure of both clusters would allow one to disentangle the effects of virgocentric infall from larger-scale motions relative to either one or more distant

Send offprint requests to: A. Schröder,

e-mail: anja@obs-nice.fr galaxy aggregates, such as the "great attractor" (Kolatt et al. 1995), or the microwave background radiation reference frame. Fornax has also been used in studies of the extragalactic distance scale (see Freedman et al. 2001, and below).

Heretofore, the H I content of the Fornax cluster galaxies has not been studied in a comprehensive way - because of the cluster's southern declination just beyond the reach of most major northern radio telescopes - (cf. Sect. 3 for references). More recent $\mathrm{H}$ I surveys of the cluster galaxies include Horellou et al. (1995) and Bureau et al. (1996). Barnes et al. (1997) conducted a shallow blind survey of the inner $8^{\circ} \times 8^{\circ}$ of the cluster; a more detailed blind survey of the whole cluster area using the Parkes Multibeam receiver (Staveley-Smith et al. 1996) is currently under way (Waugh et al. 2000).

Our main motivation in this study was to obtain an improved Tully - Fisher relation for the Fornax cluster (cf. Schröder 1995) as well as to confirm cluster membership with new $\mathrm{HI}$ radial velocities. Our sample included all 
Table 1. Observational parameters of the four runs.

\begin{tabular}{lcccc}
\hline run & May/Jun. 91 & Jan. 92 & Jun. 93 & Apr./May 94 \\
\hline system noise temperature & $40-45 \mathrm{~K}$ & $40-45 \mathrm{~K}$ & $30 \mathrm{~K}$ & $30 \mathrm{~K}$ \\
channel & 1024 & 2048 & 2048 & 2048 \\
bandwidth & $10 \mathrm{MHz}$ & $8 \mathrm{MHz}$ & $32 \mathrm{MHz}$ & $32 \mathrm{MHz}$ \\
channel spacing & $4.1 \mathrm{~km} \mathrm{~s}^{-1}$ & $1.7 \mathrm{~km} \mathrm{~s}^{-1}$ & $6.6 \mathrm{~km} \mathrm{~s}^{-1}$ & $13.2 \mathrm{~km} \mathrm{~s}^{-1}$ \\
velocity resolution (after smoothing) & $9.8 \mathrm{~km} \mathrm{~s}^{-1}$ & $7.9 \mathrm{~km} \mathrm{~s}^{-1}$ & $15.8 \mathrm{~km} \mathrm{~s}^{-1}$ & $15.8 \mathrm{~km} \mathrm{~s}^{-1}$ \\
\hline
\end{tabular}

galaxies listed in the $\mathrm{RSA}^{1}$ catalogue that lie within 5 degrees from the approximate cluster centre at $\alpha=03^{\mathrm{h}} 35^{\mathrm{m}}$ and $\delta=-35.7^{\circ}$ (basically the position of NGC 1399) and which have a radial velocity less than $2300 \mathrm{~km} \mathrm{~s}^{-1}$. To this we added those galaxies from the comprehensive catalogue of Fornax cluster galaxies by Ferguson (1989a, hereafter FCC) which he (a) judged to be either certain or possible members, and (b) classified to be of sufficiently late morphological type to suspect a detectable H I content. We also decided to reobserve some of the weaker and less well-determined $\mathrm{H}$ I lines already known from the $\mathrm{H}$ I catalogue by Huchtmeier \& Richter (1989, henceforth HR89). Finally, two possible background galaxies were added to confirm non-membership.

The final sample comprises 66 galaxies of which we detected 37 in H I. The results are presented in Sect. 3 with the description of the observations given in Sect. 2 . In Sect. 4 we analyse the distribution of $\mathrm{H}$ I content in the cluster galaxies, and in Sect. 5 we derive the Tully-Fisher relation.

\section{Observations}

The Parkes $210 \mathrm{ft}(64 \mathrm{~m})$ radio telescope was used during several sessions to observe the sample of Fornax galaxies described above. Additionally, several galaxies previously observed in the $21 \mathrm{~cm}$ line (cf. the HR89 catalogue) were reobserved to improve data quality and to allow a better comparison with previously published data. The first observing run was in late May and early June 1991, a second in January 1992, a third one in June 1993, and a fourth one in late April and early May 1994. Table 1 gives an overview of the observational parameters.

All observations were carried out in the total power mode. 10-min ON-source observations were preceded by an equal length OFF-source observation at the same declination but 10.5 min earlier in right ascension, so as to traverse the same path in geocentric coordinates during both the reference and the signal observation. To avoid having to use too many intra-cluster OFF-source positions, we attempted to use a single OFF-source observation outside the cluster area for several cluster galaxies loosely aligned along a "path" at roughly equal declination and - again spaced about $10 \mathrm{~min}$ in right ascension. It should, however,

1 A Revised Shapley-Ames Catalog of Bright Galaxies (Sandage \& Tammann 1981, 1987). be noted that a number of OFF-source positions were located well within the boundaries of the Fornax cluster.

For each galaxy such ON/OFF-observations have been repeated until the signal was unambiguous (in case of interferences) and the signal-to-noise was at least 3-5.

The dual-channel AT $21 \mathrm{~cm}$ receiver used in 1991 and 1992 had a system noise temperature of the order of 40-45 K. In 1993 and 1994 a new receiver with a system noise temperature of about $30 \mathrm{~K}$ was used. In 1991 a 1024-channel autocorrelator served as the backend. It was split into two banks of 512 channels each which detected the two independent polarisations. A bandwidth of $10 \mathrm{MHz}\left(\approx 2100 \mathrm{~km} \mathrm{~s}^{-1}\right)$ with a central frequency corresponding to a heliocentric radial velocity of $1300 \mathrm{~km} \mathrm{~s}^{-1}$ was used for all galaxies regardless of the availability of a known radial velocity. Beginning in 1992 a new autocorrelator (technically quite similar to the correlators in use at the Australia Telescope Compact Array in Narrabri) was available with 2048 channels, which were also split into 2 banks of 1024 channels and $8 \mathrm{MHz}$ bandwidth each. Once, in January 1992, this new autocorrelator suffered a processor failure and data for a single 24-hour period were again taken with the old autocorrelator.

The autocorrelator setup was changed in 1993 to accommodate other parallel programs to be reported elsewhere. The bandwidth was changed to $32 \mathrm{MHz}$ with 1024 channels in each bank. In 1994 we used a split into 4 banks of 512 channels each with an IF offset of $25 \mathrm{MHz}$ for the third and fourth bank of the autocorrelator to also cover a higher velocity range out to $12000 \mathrm{~km} \mathrm{~s}^{-1}$. This information came basically free of cost, since banks 1 and 3 as well as 2 and 4 were fed by the same polarisation output from the receiver, i.e., would have differed only by the quantization noise which was practically undetectable. No serendipitous signals, however, showed up in this higher velocity range for Fornax galaxies.

In all cases the two different polarisations were averaged during data reduction. Fitted spectral baselines consisting of a polynomial of moderate order added to a sine function with a period equal to that of the standing wave pattern of the Parkes telescope $(5.8 \mathrm{MHz})$ were subtracted to form the final spectra.

The online control program automatically corrected for the zenith angle dependence of the telescope sensitivity. The primary flux calibration was obtained by measuring standard sources from the Parkes catalogue (Wright \& Otrupcek 1990) at the beginning of each observing session; 
it was stable to within $\approx 10 \%$. As an added check of system performance, secondary $\mathrm{H}$ i flux calibrators chosen from the new compilation of $\mathrm{HI}$ data for RSA galaxies by Richter et al. (1994, private communication) were observed from time to time. Based on those data the internal consistency of the flux scale is judged to be better than $15 \%$.

\section{Results}

We present our results in this section as a table of the $\mathrm{H}$ I measurements, notes on individual galaxies and a comparison with other measurements. The H I spectra of the detected galaxies are shown in the Appendix. It should be noted that the number of Hanning smoothing operations for the displayed spectra varies from 1 to 4 depending on the overall signal-to-noise ratio.

\subsection{The new HI data}

In Table 2 we present the averaged data of the four runs (in general a weighted mean) together with some parameters from the FCC. The Table columns are as follows.

Column 1a: Galaxy identification according to the FCC.

Column 1b: Other galaxy identification: NGC, IC, ESO in this order of preference.

Column 2: Coordinates in J2000 equinox.

Column 3: Morphological type from the FCC. Where the galaxy was not in FCC, $\mathrm{RC} 3^{2}$ values have been taken.

Column 4: $B$-band magnitude from the FCC. Where the galaxy was not in FCC, RC3 values have been taken.

Column 5: Decimal logarithm of the diameter, $\log D$, in units of 0.1 at the 26.5th $B$-band isophote from the FCC. For non-FCC members we have taken $\log D_{25}$ from the RC3 and added 0.05, which is the mean difference between FCC and RC3 values.

Column 6: Axial ratios, $\log R$, from Lauberts \& Valentijn (1989). For galaxies with no entry we have taken the axial ratios from Paturel et al. (2000; cf. the LEDA ${ }^{3}$ database). They are indicated with a superscript $a$. For one galaxy (superscript $b$ ) the axial ratio comes from MacGillivray et al. (1988), and for two galaxies no axial ratios could be found in the literature and our own measurements are given (superscript $c$ ).

Column 7a: Weighted mean heliocentric H I radial velocity in $\mathrm{km} \mathrm{s}^{-1}$ taken at the midpoint of the H I profile at the $20 \%$ level. The optical convention $v=c\left(\lambda-\lambda_{\circ}\right) / \lambda_{\circ}$ is used.

Column 7b: Error of the radial velocity, calculated according to Fouqué et al. (1990): $\sigma(v)=4 R^{0.5} P^{0.5} \mathrm{~s}^{-1}$ where $R$ is the resolution in $\mathrm{km} \mathrm{s}^{-1}$ (1.2 times the channel spacing), $P$ is the steepness of the edges of the profile

\footnotetext{
2 Third Reference Catalogue of Bright Galaxies (deVaucouleurs et al. 1991).

3 Lyon-Meudon Extragalactic DAtabase; http://leda.univ-lyon1.fr
}

$\left(P=\left(\Delta v_{20}-\Delta v_{50}\right) / 2\right), s$ is the signal-to-noise, i.e., peak $S_{\mathrm{h}}$ over rms noise level.

Column 8a: Velocity width in $\mathrm{km} \mathrm{s}^{-1}$ of the H I profile measured at the $50 \%$ level of the peak intensity, $\Delta v_{50}$, corrected for instrumental broadening: no correction for $0<R \leq 8 \mathrm{~km} \mathrm{~s}^{-1},-3 \mathrm{~km} \mathrm{~s}^{-1}$ for $8<R \leq 16 \mathrm{~km} \mathrm{~s}^{-1}$, $-5 \mathrm{~km} \mathrm{~s}^{-1}$ for $16<R \leq 32 \mathrm{~km} \mathrm{~s}^{-1},-7 \mathrm{~km} \mathrm{~s}^{-1}$ for $32<R$. The smallest line width of the four runs has been selected (since there is no known mechanism that would artificially decrease the line width).

Column 8b: Velocity width in $\mathrm{km} \mathrm{s}^{-1}$ of the H I profile measured at the $25 \%$ level of the peak intensity, $\Delta v_{25}$, corrected for instrumental broadening (cf. Col. 6a).

Column $8 c$ : Velocity width in $\mathrm{km} \mathrm{s}^{-1}$ of the H I profile measured at the $20 \%$ level of the peak intensity, $\Delta v_{20}$, corrected for instrumental broadening (cf. Col. 6a).

Column 9a: Weighted mean $\mathrm{H}$ I flux, which is the line integral in $\mathrm{Jy} \mathrm{km} \mathrm{s}^{-1}$, uncorrected for finite beam size.

Column 9b: The error of the HI flux is the combined error for the measurement of the profile and the flux calibration: $\sigma=\left(\sigma^{2}(A)+(0.1 A)^{2}\right)^{0.5}$ with $\sigma(A)=$ $5 R^{0.5} A^{0.5} \mathrm{~h}^{0.5} \mathrm{~s}^{-1}$ (according to Fouqué et al. 1990) where $A$ is the line integral and the other parameters as described for Col. 5b.

Column 10: Weighted mean H i peak flux $S_{\mathrm{h}}$ in Jy.

Column 11: Calculated H I mass in $10^{8} d_{20}^{2} M_{\odot}$ using $M_{\mathrm{HI}}=2.365 \times 10^{5} d^{2} S=948 \times 10^{5} S d_{20}^{2} M_{\odot}$, where $S$ is the H I flux from Col. 9a, and the distance is $d=20 d_{20} \mathrm{Mpc}$ (cf. Sect. 4.3). No H I masses were computed for background detections.

Column 12: The rms noise level $S_{\text {rms }}$ over the region used to fit a baseline, in $10^{-3} \mathrm{Jy}$. The smallest values of the four runs are given here.

Column 13: The runs when the galaxy was observed.

Column 14: Notes: the search interval for the possible background galaxy FCC B37 is given. A star denotes interferences in the spectra which could have prevented us from detecting the line. It is indicated at the bottom of the table if a velocity is known for that object. A hash sign indicates if the smallest line width has been discarded because of either a very low $S / N$ of about three or a large correction for instrumental broadening $\left(\geq 7 \mathrm{~km} \mathrm{~s}^{-1}\right)$. A dagger means that the diameter has been derived from RC3 values.

\subsection{Notes on individual objects}

FCC 2 was classified as a cluster member by Ferguson (1989a) but we have shown it to be a background spiral at $v=4540 \mathrm{~km} \mathrm{~s}^{-1}$.

FCC 35 shows two unusual-looking peaks. It was confirmed to be at $1800 \mathrm{~km} \mathrm{~s}^{-1}$ by follow-up observations made at Parkes by Staveley-Smith (1994, private communicaion) and has more recently been observed with the ATCA (Australia Telescope Compact Array) by Putman et al. (1998), who found that one of the peaks is caused by a nearby intergalactic H I cloud, and the other, as presented here, by the galaxy proper. 
Table 2. Final adopted results of H I observations of Fornax cluster galaxies.

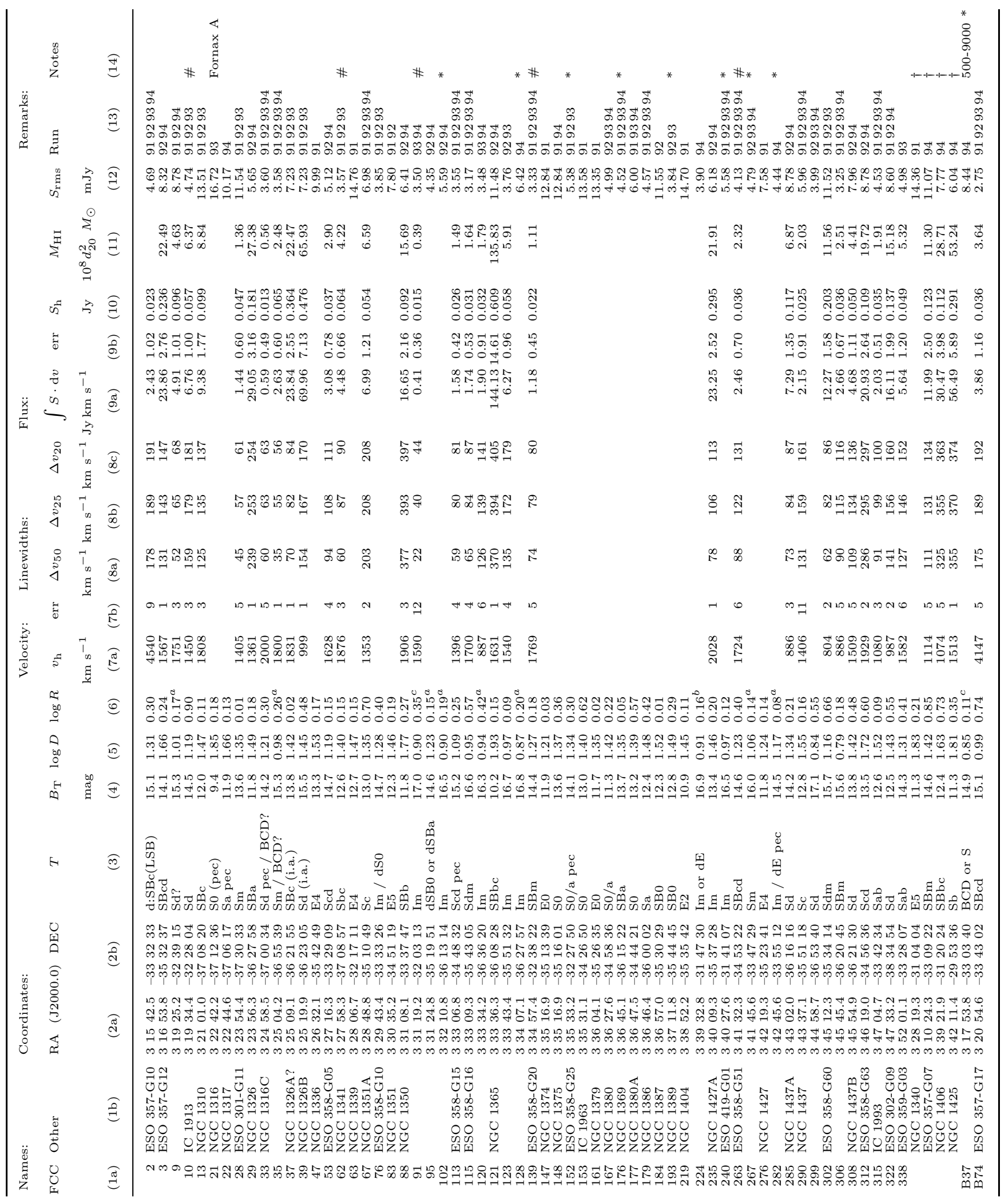

Notes: *: FCC 102: no $v$, small interf. at $\sim 1200$ and FCC 121 at $\sim 1650$; FCC 128: no $v$, small interf. at $\sim 1250$; FCC 152: large interf. at $\sim 1200$ close to $v=1465$ (Richter \& Sadler 1985); FCC 176: large interf. at $\sim 1250$ close to $v=1468$ (Richter \& Sadler 1985); FCC 193: small interf. at $\sim 800$ and $\sim 1200, v=989$, should not be affected; FCC 240: no $v$, large interf. at $\sim 1250$; FCC 267: no $v$, large interf. at $\sim 1250$; FCC 282: small interf. at $\sim 1250$ close to $v=1295$ (da Costa et al. 1991); FCC B37: no $v$, large interf. at $\sim 1250$; \#: the second smallest line width has been adopted. $\dagger: \log D$ is derived from RC3 values. Col. 5 (log $R$ ): superscript $a$ : axial ratio from Paturel et al. (2000); $b$ : axial ratio from MacGillivray et al. (1988); $c$ : axial ratio measured by the authors. 
FCC 121 (NGC 1365) was used as an intra-cluster check of system performance by separately reducing each 10-min observation, in each polarisation band, of this galaxy. Note that only the final averaged data are given in the table.

FCC 306 lies in the beam of FCC 308 and has been confirmed in a FLAIR observation with $v=915 \pm 15 \mathrm{~km} \mathrm{~s}^{-1}$ using emission lines (Schröder 1995).

FCC 338 was classified as S0 in FCC but seems more likely to be an Sab as given in RC3.

FCC B74, the brightest background spiral galaxy in Ferguson's classifications, has been confirmed with $v=4147 \mathrm{~km} \mathrm{~s}^{-1}$.

\subsection{Comparison with earlier data}

Galaxies in the Fornax cluster have been previously surveyed for H I content by - among others - Fouqué et al. (1990), Mathewson et al. (1992), Horellou et al. (1995), Bureau et al. (1996), and Barnes et al. (1997). For a detailed listing of references before 1989 the reader is referred to the HR89 catalogue.

In general, the agreement of our H I radial velocities with published values is excellent with deviations never exceeding the stated - usually internal - errors. However, H I fluxes vary considerably with differences more often than not larger than the quoted relative errors (usually $10-15 \%$, cf. Sect. 2). Varying signal-to-noise ratios for the weaker H I spectra have a significant impact also on the measured H I line widths. During our own observations, we therefore attempted to improve upon the weaker signals rather than observing a larger number of objects. Yet, not all line widths are of the same quality.

We have compared the line widths with published data in the literature: Bottinelli et al. (1990), Mathewson et al. (1992), and Bureau et al. (1996) give H I line widths for Fornax galaxies. The agreement is within the errors for both $20 \%$ and $50 \%$ line widths except in the case of the $20 \%$ line width of Bureau et al. which show a mean deviation of $10 \pm 3 \mathrm{~km} \mathrm{~s}^{-1}$ (for 18 galaxies in common). The comparison with the other sources indicates that their line widths are too broad by $\sim 7 \mathrm{~km} \mathrm{~s}^{-1}$ (after correction for instrumental broadening).

\section{The $\mathrm{H}$ I content of the Fornax cluster}

In this section we examine the distribution of $\mathrm{H} \mathrm{I}$ in the Fornax cluster based on our observations. We first describe the galaxies detected in $\mathrm{H}$ I and then compare their distribution with that of the galaxies not detected before comparing their $\mathrm{HI}$ masses with galaxies in other environments.

\subsection{The HI detections}

Our data represent some of the deepest observations ever made of Fornax cluster galaxies in H I. We detected 37 of the 66 galaxies, 12 of them for the first time (and two

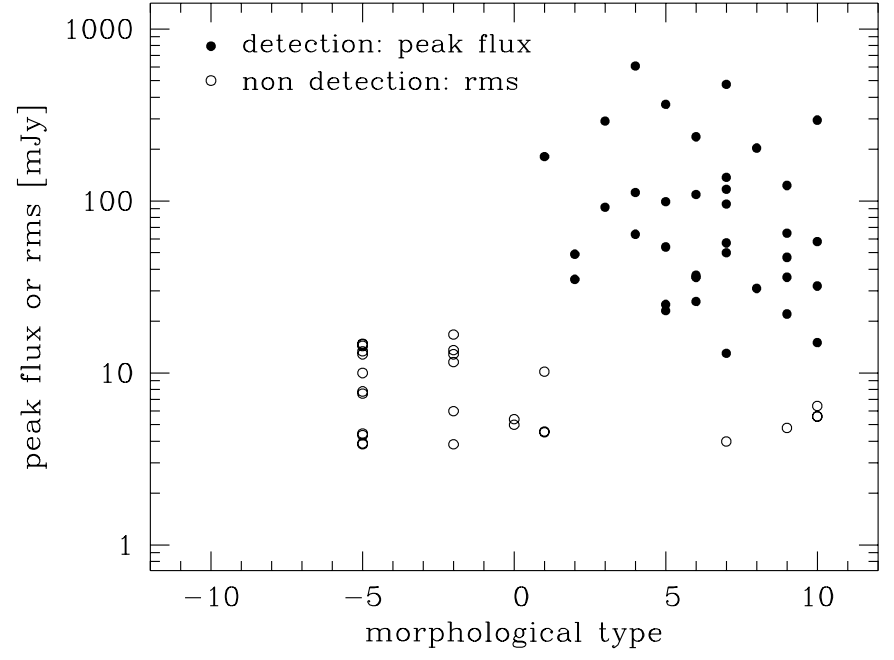

Fig. 1. Distribution of morphological type for detections (filled circles: peak flux is given) and non-detections (open circles), which are represented through the rms of the $\mathrm{H}$ i spectrum.

being background galaxies). Figure 1 shows the morphological types of our sample (with the numerical coding as in RC3): non-detections are represented through the standard deviation of the noise (rms) of their H I spectra (open circles), whereas the peak flux is given for the detected galaxies. Only a few spiral galaxies were not detected, in particular three out of four Sa galaxies. Four of the undetected late-type galaxies are affected by interference at $v \simeq 1250 \mathrm{~km} \mathrm{~s}^{-1}$, which may have prevented the detection of the galaxy in its vicinity (no velocities are known from the literature). The fifth galaxy (FCC 299) has an optical velocity of $2051 \pm 9 \mathrm{~km} \mathrm{~s}^{-1}$ (Schröder 1995). A marginal detection at this velocity is possible but would require at least two more hours of observation to be confirmed.

No elliptical or S0 galaxies $(T=-2)$ were detected. We have assigned $T=-5$ to the dwarf spheroidal and $\mathrm{Im} / \mathrm{dE}$ galaxies since these classifications indicate early type galaxies.

\subsection{The distribution of $\mathrm{HI}$ detections}

In the following discussions we have not included the two galaxies with $\mathrm{HI}$ detected at high velocity (FCC 2 and FCC B74) as they are not cluster members.

The present paper gives the most comprehensive list of H I detections in the Fornax cluster to date. In Fig. 2 we show the distribution of FCC galaxies in the sky, with the $\mathrm{HI}$ detections as filled circles and the non-detections as open circles. Note that our sample includes four galaxies outside the FCC as well as three background galaxies of which two are not in the FCC main catalogue. To quantify, we plot in Fig. 3 the numbers of galaxies in radial annuli of equal area for the total sample and the galaxies with and without $\mathrm{H}$ I detections.

Both Figs. 2 and 3 show that the galaxies with HI detections are evenly distributed across the cluster, whereas those without any detected $\mathrm{H}$ I are much more 


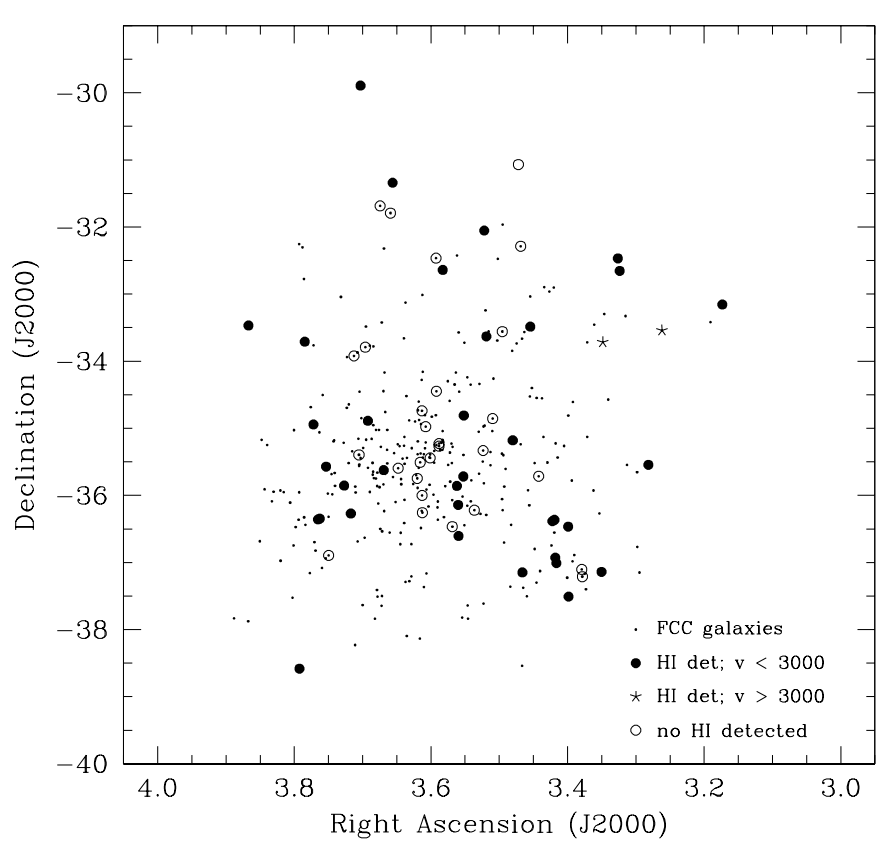

Fig. 2. The distribution of FCC galaxies (dots), H i detections of the present work (filled circles) and non-detections (open circles) in J2000 coordinates. Stars indicate H I detections of background galaxies.

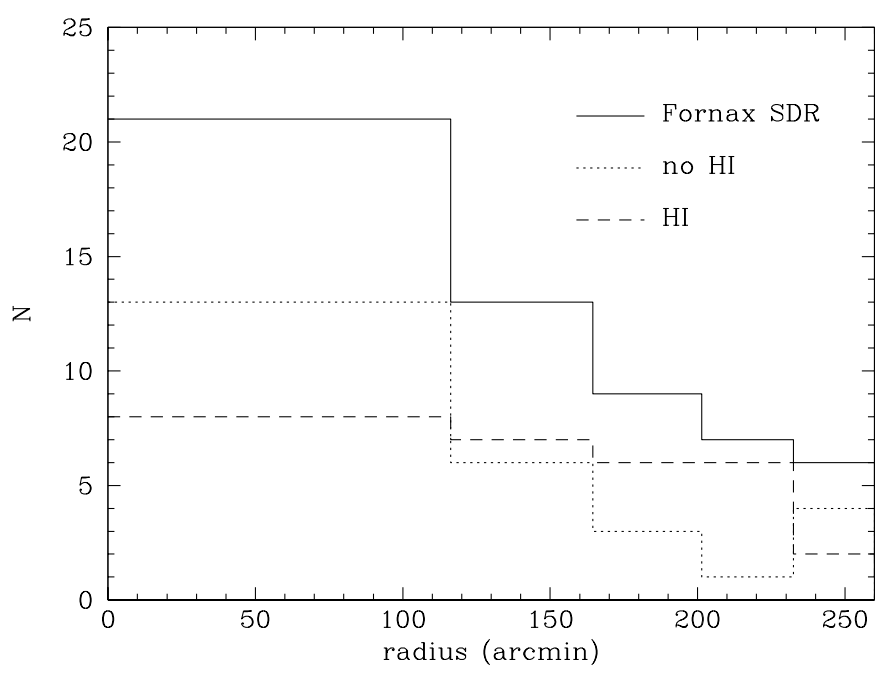

Fig. 3. The distribution of our total sample, detections (dashed line) and non-detections (dotted line) in equal area bins as a function of distance from the cluster centre.

concentrated towards the cluster centre. This is to a large extent a reflection of the usual density-morphology relation: the ratio of early to late-type galaxies increases rapidly towards the cluster core. In Fig. 3 the distribution of galaxies with detected $\mathrm{H} \mathrm{I}$ is consistent with a constant surface density as though these galaxies were not aware of the cluster, although this is partly due to the binning: see the cumulative distribution in Fig. 4.

In Fig. 4 we plot the same data as cumulative distribution functions, also including cluster members listed in the optical FCC catalogue. We can now assess the significance of the difference in the distributions. The radial

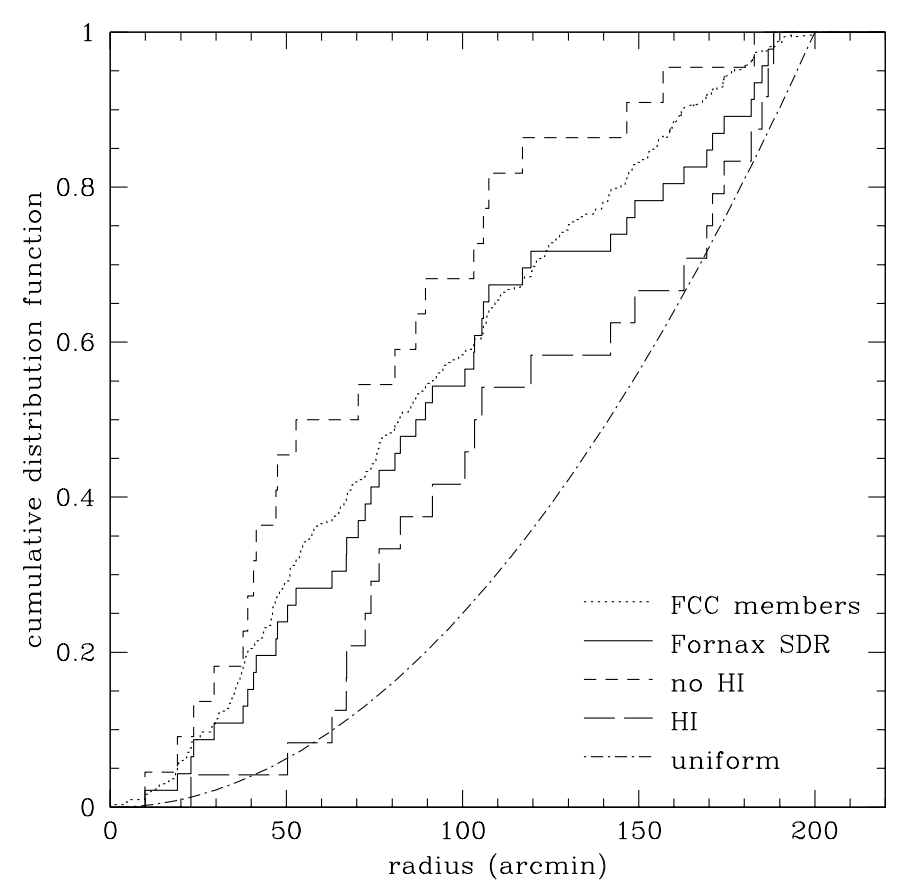

Fig. 4. Cumulative radial distribution of our total sample (solid line) compared to the distribution of all cluster members in the optical FCC sample (dotted line). The subsamples of our galaxies with and without any detected $\mathrm{HI}$ are indicated by long and short dashed lines respectively. A uniform, constant-density distribution is also shown for comparison.

distribution of our total sample is not significantly different from that of the FCC members: they differ at a Kolmogorov-Smirnov (KS) significance of only $15 \%$. There is a significant difference in the radial distributions of the $\mathrm{HI}$ and non-HI samples: they differ at a KS significance of $97 \%$ with the non-detected galaxies being much more concentrated towards the cluster centre. Interestingly, the distribution of the $\mathrm{H}$ I detected galaxies is also more centrally concentrated than a uniform distribution (dashdotted curve in Fig. 4) at a KS significance of $94 \%$. The H I-rich galaxies are not distributed as a uniform sheet but clearly show signs of being concentrated towards the cluster although at larger radii than the non-detected galaxies.

We also compared the velocity distribution of the cluster galaxies detected in $\mathrm{HI}$ with published velocities of the other cluster members in our sample. The 35 cluster galaxies detected in $\mathrm{H}$ i have a mean velocity of $1480 \pm 60 \mathrm{~km} \mathrm{~s}^{-1}$ and the 26 members not detected in $\mathrm{H}_{\mathrm{I}}$ with published velocities have a mean velocity of $1460 \pm 70 \mathrm{~km} \mathrm{~s}^{-1}$. There is no significant difference in these velocity distributions.

\subsection{The HI masses}

Estimates of the HI mass depend on the cluster distance, which is currently under discussion. There are Cepheid distances available for three Fornax galaxies: NGC 1365 at 18.6 Mpc (Madore et al. 1999), NGC 1326A at $18.7 \mathrm{Mpc}$ (Prosser et al. 1999), and NGC 1425 at 22.2 Mpc (Mould et al. 2000). Drinkwater et al. (2001a) 


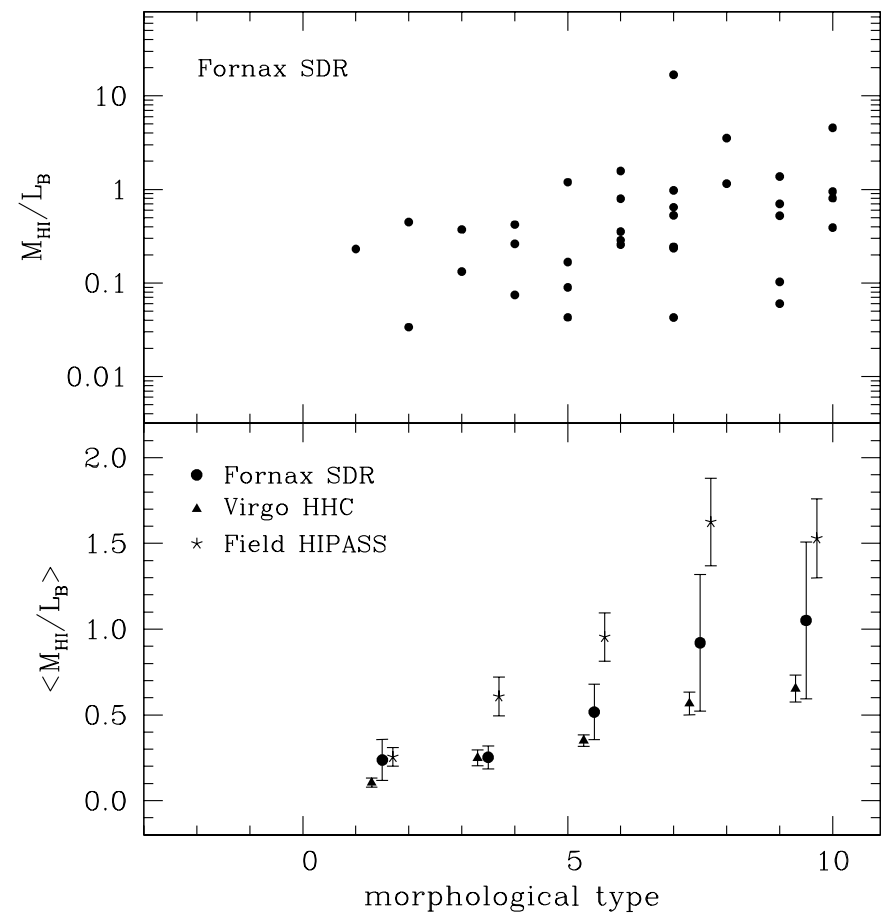

Fig. 5. The H I mass-to-blue light ratio versus morphological type for our sample. The upper panel shows the distribution of the sample in logarithmic scale, the lower panel shows the binned data in comparison with the Virgo cluster (triangles) and with the field (stars). For clarification the points are slightly offset in types.

have shown that NGC $1326 \mathrm{~A}$ is part of a subcluster in front of the main cluster (see Sect. 5) and they also argue that NGC 1365 is in front of the cluster core. They therefore propose - as did Mould et al. (2000) - that the mean of the three distances, $20 \mathrm{Mpc}$, be adopted for the Fornax cluster. We note that there is still no consensus in the literature on this matter, and for this paper we parameterise the distance as $d=20 d_{20} \mathrm{Mpc}$.

The Hi masses for our galaxies are calculated using $M_{\mathrm{HI}}=2.37 \times 10^{5} d^{2} S=948 \times 10^{5} S d_{20}^{2} M_{\odot}$, where $S$ is the Hi flux integral in $\mathrm{Jy} \mathrm{kms}^{-1}$. These distanceparameterised masses are listed in Table 2 in units of $d_{20}^{2} M_{\odot}$.

NGC $1365(T=4)$ has the largest H I mass in Fornax and is one of the most H I-massive galaxies known. The second most H I-massive galaxy in Fornax, NGC 1326B $(T=7)$, is one member of an interacting pair. Its H I massto-light ratio is extremely large, presumably due to its interactions (cf. Fig. 5). Its companion, NGC 1326A $(T=5)$, shows no unusual parameters.

NGC 1316C has the lowest H I mass for the Sd galaxies. It is a close neighbour of FCC $35(T=9)$ which is associated with an H I cloud (Putman et al. 1998). This H I cloud may have been stripped from NGC 1316C by a close encounter with FCC 35, which has an H I mass consistent with its morphological type.
The distance-independent $\mathrm{H}$ I mass-to-blue light ratio $M_{\mathrm{HI}} / L_{B}$ is shown in the upper panel of Fig. 5 in logarithmic scale. We have binned the data in pairs of morphological types for better statistics $(1-2,3-4,5-6,9-10)$ and calculated the mean. The result is shown in the lower panel of Fig. 5. NGC 1326B with its extreme $M_{\mathrm{HI}} / L_{B}$ has been excluded from this mean.

In comparison we show the means of galaxies in the Virgo cluster (triangles), as derived from data obtained with the Arecibo telescope (Helou et al. 1981; Helou et al. 1984; Hoffman et al. 1987; Hoffman et al. 1989; for simplicity we shall call this the HHC data, as in Helou, Hoffman and collaborators). For the calculation of the mean we have again excluded an unusually large $M_{\mathrm{HI}} / L_{B}$ (for $T=3$ ) in this data-set. Despite the larger errors for the Fornax sample (due to small sample statistics) we can see that the Virgo galaxies, well-known for their H I depletion, have systematically lower $M_{\mathrm{HI}} / L_{B}$ ratios. The mean $M_{\mathrm{HI}} / L_{B}$ for the Fornax sample is $0.68 \pm 0.15$ whereas that of the Virgo sample is $0.45 \pm 0.03$. The means differ at the $85 \%$ confidence level according to the T-test.

Finally, we also show the means from data obtained in the H I Parkes All-Sky Survey (HIPASS) of bright field galaxies in the southern hemisphere (Marquarding 2000; see also Staveley-Smith et al. 1996 for a description of the survey). The H I selected field galaxies (indicated with stars) show systematically larger $M_{\mathrm{HI}} / L_{B}$ ratios than the galaxies in either cluster. The mean $M_{\mathrm{HI}} / L_{B}$ for the field sample is $1.15 \pm 0.10$ which is larger than the Fornax value at the $98 \%$ confidence level and larger than the Virgo value at a confidence level greater than $99.9 \%$ (both according to the T-test).

Galactic extinction corrections have been applied to the HIPASS sample; in case of Fornax and Virgo we assume the Galactic extinction to be zero or negligible. Since the HIPASS sample does not have inclinations for all galaxies we have not corrected the blue luminosities for any of the samples for internal absorption. A correction for internal absorption will lower the mean value of $M_{\mathrm{HI}} / L_{B}$. The HIPASS sample is limited by peak flux and therefore biased against largely inclined galaxies. However, the correction in this case would be smaller than for the cluster samples with their more evenly distributed inclinations and the difference between field and cluster galaxies will be increased.

\subsection{HI deficiency}

Solanes et al. (1996, hereafter SGH) have shown that the $\mathrm{H}$ I content of isolated spiral galaxies not only depends on morphological type but also shows a tight linear relation with the linear optical diameter. We can therefore compare the actual observed H I mass as derived from our H I fluxes with the expected value for a galaxy unaffected by environmental conditions and of the same diameter and morphological type. Using the tabulated coefficients for the expectation value of the logarithm of the H I mass, 
$\log \widehat{M}(T, D)$, from SGH, we have calculated the H I deficiencies for our galaxies as

$\mathrm{HI}_{\mathrm{def}}=\log \widehat{M}_{\mathrm{HI}}\left(T^{\mathrm{obs}}, D_{\mathrm{opt}}^{\mathrm{obs}}\right)-\log M_{\mathrm{HI}}^{\mathrm{obs}}$,

where the diameters are given in $\mathrm{kpc}$ and the $\mathrm{H}$ I masses in solar units. We have used the diameters from the FCC (measured at the 26.5th isophote), and corrected RC3 diameters for 3 galaxies outside of the FCC by adding the mean difference of 0.05 . Since SGH have used $\mathrm{UGC}^{4}$ diameters, we have then adjusted the conversion given by Horellou et al. (1995) to compute UGC from our FCC diameters:

$\log \left(D_{\mathrm{UGC}}+0.3\right)=1.0173 \log D_{25}$.

Because of the additional correction for diameter dependence, we would expect the $\mathrm{HI}$ deficiencies to have a smaller internal scatter and be a better indicator for variations in $\mathrm{HI}$ content than the $M_{\mathrm{HI}} / L_{B}$ values. However, in addition to the (sometimes large) errors in observed $\mathrm{H} \mathrm{I}$ flux, diameter and morphological type, both the H I mass and the linear diameters are distance dependent, which increases the scatter in the $\mathrm{HI}$ deficiencies again. While the same distance can be used for a small nearby cluster as Fornax, the distances to the field galaxies used for the expectation value by SGH depend on the Hubble constant and show errors due to peculiar velocities. Solanes et al. (2001) therefore emphasise that H I deficiencies are only meaningful in a statistical sense.

Expectation values of $\mathrm{H}$ I masses are only well determined for types $T=1-5$. As indicated by Solanes et al. (2001), we have calculated the expectation values of $\mathrm{HI}$ masses for the later types $(T=6-10)$ using the coefficients for $T=5$. Though the variation in the coefficients with morphological type are small (cf. Haynes \& Giovanelli 1984), we want to emphasise that H I deficiencies for types (much) later than 6 are less reliable.

Figure 6 shows the H I deficiencies of all our Fornax galaxies with $\mathrm{H}$ I measurements. The scatter is large and the number of galaxies for each morphological type is small. We have therefore derived a mean $\mathrm{H}$ I deficiency for the types $T=1-5$ together: $\left\langle\mathrm{HI}_{\mathrm{def}}\right\rangle=0.35 \pm 0.13$. Including galaxies with $T=6$, we find the similar value of $\left\langle\mathrm{HI}_{\mathrm{def}}\right\rangle=0.38 \pm 0.09$. If we assume that the deficiency is reliably defined for the later types as well, we find $<\mathrm{HI}_{\text {def }}>=0.30 \pm 0.07$, which is close to the other values. These results show a significant if moderate $\mathrm{H}$ I deficiency for the Fornax cluster, independent of our $M_{\mathrm{HI}} / L_{B}$ calculations above. Our deficiency value is consistent with that of Horellou et al. (1995) who derived a deficiency of $0.43 \pm 0.55$, with a much larger uncertainty.

We have calculated $\mathrm{HI}$ deficiencies for the HIPASS sample as well, using RC3 diameters where available (for 106 out of 136 galaxies). The mean deficiencies for all types together as well as for each type separately are consistent with zero (e.g., $0.05 \pm 0.03$ for $T=1-6$ ), indicating that the sample, though H I selected and not optically selected,

\footnotetext{
${ }^{4}$ Uppsala General Catalog of Galaxies (Nilson 1973).
}

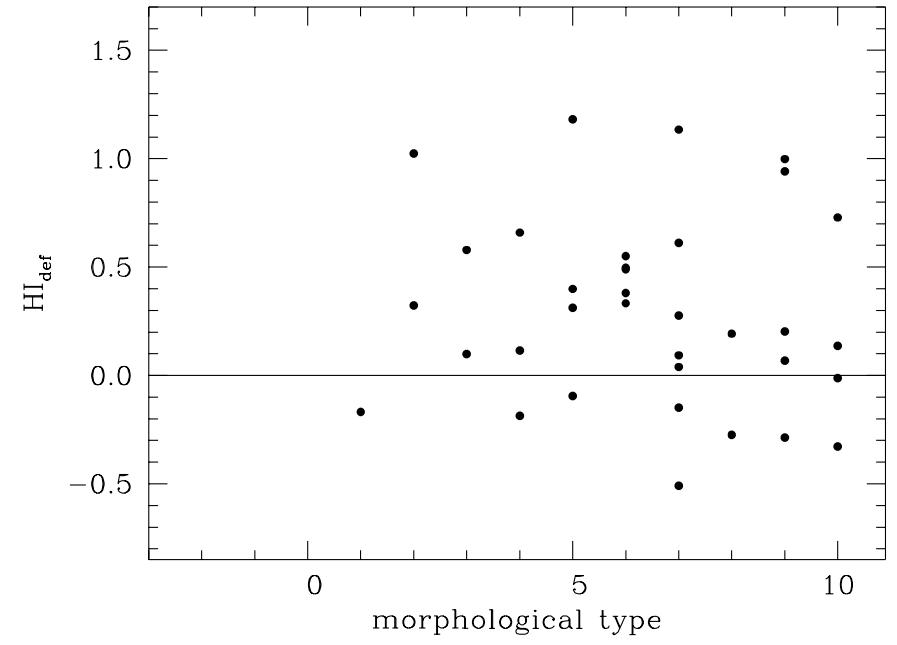

Fig. 6. The H I deficiency parameter versus morphological type for our sample. The expected H I masses for galaxies with $T \geq 6$ were calculated using the relationship for galaxies with $T=5$.

is representative for the field. The scatter for $T=1-6$ is 0.24 and comparable to the scatter of 0.23 quoted by SGH for their field galaxies.

\subsection{Discussion}

Environmental effects on the H I content of spiral galaxies have been discussed at length in the literature (e.g., Solanes et al. 2001; Abadi et al. 1999; van Gorkom 1996; Giovanelli \& Haynes 1985; and many others). There are two main mechanisms that can strip galaxies of their gas content: ram-pressure stripping by interaction with hot intra-cluster gas, and tidal interactions with other galaxies. The second mechanism can also strip stars off the outer parts of a galaxy. Other mechanisms are viscous stripping and thermal conduction. Rich galaxy clusters, like Virgo (Solanes et al. 2001; Cayatte et al. 1994; Guhathakurta et al. 1988; Hoffman et al. 1988; to name only a few) or Coma (Bravo-Alfaro et al. 2000; Bothun et al. 1984) have large X-ray luminosities, and galaxies in the inner part of the clusters show definite signs of loss of $\mathrm{H}$ I gas due to ram-pressure stripping.

Fornax is a small cluster but with a high central galaxy density. Its X-ray luminosity is much lower than that of the Virgo cluster (about 2 orders of magnitude) with a significant detection only in the core of the cluster (Killeen \& Bicknell 1988; Jones et al. 1997). Within the core region however (a projected radius of about 20 arcmin) the mean gas density is of order $10^{-3} \mathrm{~cm}^{-3}$ (Jones et al.) so pressure stripping will be effective with timescales of $10^{9} \mathrm{yrs}$ according to the conservative estimates of Ferguson \& Binggeli (1994). There is also direct evidence for stripping in some of the central Fornax galaxies from their morphologies, e.g. NGC 1404 (Jones et al. 1997). The effect of pressure stripping is harder to calculate at larger radii, but it is presumably much lower. In a large sample of galaxies from different clusters (most well-outside the core region), Solanes et al. (2001) found no apparent 
relationship between (strong) X-ray luminosity and H I deficiency in individual galaxies. (However the fraction of gaseous late-type spirals in the centre of rich clusters may be reduced in favour of lenticulars that have no or only little gas content.)

Our Fornax data demonstrate a non-zero H I deficiency parameter as well as a mean $M_{\mathrm{HI}} / L_{B}$ ratio significantly lower than that found for a sample of field galaxies. This is for a sample of galaxies that extends well beyond the core where we might expect pressure stripping to be important. This result appears to conflict with previous nondetections of any strong H I deficiency in Fornax (Horellou et al. 1995; Bureau et al. 1996). In the former case the sample actually measured was very small (only 6 new measurements of cluster galaxies), which resulted in a large uncertainty, and they were mostly earlier types where the depletion is weaker. In the case of the Bureau et al. result the conclusion was based on a comparison with galaxies in the Ursa Major cluster. We note that the Fornax cluster is not significantly denser than Ursa Major if the central core region is excluded and that we might therefore expect them to have similar H I deficiencies. Our use of a large sample and our comparison with a genuine field sample from the HIPASS data (Marquarding 2000) has allowed us to detect the H I deficiency in the Fornax cluster.

As we describe above, a number of mechanisms have been proposed that could explain the H I deficiency of Fornax cluster galaxies. Most of the galaxies we detected in $\mathrm{HI}$ are well beyond the cluster core where the pressure of the hot X-ray gas is high enough for ram-pressure stripping. However, as discussed by Solanes et al. (2001), this mechanism is still viable if the galaxies are on radial orbits in the cluster. Our sample is too small for a detailed analysis of the orbits, but we can make a simple comparison of the kinematics of the $\mathrm{H}$ I-deficient galaxies $\left(\mathrm{HI}_{\mathrm{def}}>0.3, n=17\right)$ with the rest of the $\mathrm{H}$ I detected galaxies. The $\mathrm{H}$ I-deficient galaxies have a smaller velocity dispersion $\left(247 \pm 44 \mathrm{~km} \mathrm{~s}^{-1}\right)$ than the other galaxies $\left(391 \pm 67 \mathrm{~km} \mathrm{~s}^{-1}\right)$ with the difference significant at the $93 \%$ confidence level. Splitting the sample according to $M_{\mathrm{HI}} / L_{B}$ ratio gives very similar results. This difference in velocity dispersions is consistent with the deficient galaxies having more radial orbits than the other galaxies as was shown for a composite cluster sample by Solanes et al. (2001). One further process that will contribute to H I depletion is the conversion of neutral gas into stars. Our optical measurements (Drinkwater et al. 2001b) have shown that there is an excess of star formation in Fornax cluster galaxies at similar distances to most of our $\mathrm{H} \mathrm{I}$ detections. Although the corresponding gas depletion timescales are long (of order $10^{10} \mathrm{yrs}$ ), this does show that the cluster environment can influence the gas content of galaxies even at these large distances.

\section{The Tully-Fisher relation}

During our observing programme we observed all spiral galaxies in the FCC with long integration times. In

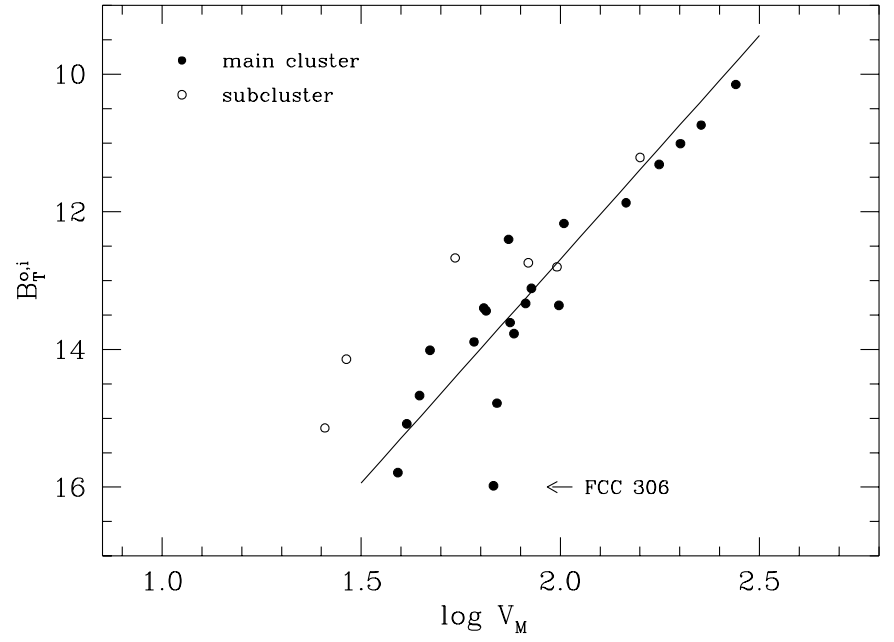

Fig. 7. The Tully-Fisher relation for the Fornax cluster. Open circles denote galaxies which seem to belong to a subcluster. The linear regression is derived for the main cluster only and without FCC 306.

combination with the multi-colour multi-aperture data obtained by one of us (Schröder \& Visvanathan 1996; Schröder 1995) this comprehensive sample enables us to establish the Tully-Fisher relation for all inclined spiral galaxies $\left(i \geq 45^{\circ}, 1 \leq T \leq 9\right)$ in the Fornax cluster. We have taken the parameters as well as the corrections as described in Schröder (1995): morphological types are from the FCC, axial ratios are mainly from Lauberts \& Valentijn (1989, cf. notes to Table 2), $q_{0}$ to calculate inclinations are from Heidmann et al. (1971), and internal absorptions have been corrected according to the RC3. We have taken the $20 \%$ line widths from Table 2 (which are corrected for instrumental broadening), and applied a correction for z-motion and turbulences of $-12.6 \mathrm{~km} \mathrm{~s}^{-1}$ as explained in Richter \& Huchtmeier (1984). For the TullyFisher relation we use the maximum rotational velocity $V_{\mathrm{M}}=\Delta v_{20}^{\mathrm{c}}(2 \sin i)^{-1}$. Adopting a slope of -6.50 from the Virgo cluster (Schröder 1995) where we have better statistics, we find

$B_{\mathrm{T}}^{\mathrm{o}, i}(F)=-6.50 \cdot\left[\log V_{\mathrm{M}}-2.0\right]+(12.56 \pm 0.18)$

with a scatter of $0.84 \mathrm{mag}$.

Excluding the faintest galaxy from the relation, FCC 306 (cf. Fig. 7), we find a scatter of 0.71, which is considerably smaller. No obvious explanation for such a deviation could be found: this Sm galaxy has a strong H I signal, and the velocity has been confirmed with an optical measurement (Schröder 1995). Its 20\%-width is possibly smaller by $\sim 15 \mathrm{~km} \mathrm{~s}^{-1}$ (Waugh, priv. comm., using HIPASS data) which would result in a scatter of 0.81 . Its $\mathrm{HI}$ mass and $M_{\mathrm{HI}} / L_{B}$ is consistent with other galaxies of this type. The optical data is good (four apertures covering a large range in radius of the galaxy) and the total magnitude is comparable to the magnitude given by Lauberts \& Valentijn (1989). The axial ratio is confirmed by Loveday (1996). However, we believe that its unusually large effect on the scatter of the Tully-Fisher relation justifies 
sufficiently its exclusion from here on from the relation. We assume that a combination of errors in the various parameters accounts for its unusual high deviation from the Tully-Fisher relation. However, another explanation would be that this galaxy lies off the main Tully-Fisher relation because it actually lies behind the main cluster and and is falling into it. This argument is supported by the very compact morphology of FCC 306: it has the smallest angular size and highest surface brightness of the starforming dwarfs in the sample of Drinkwater et al. (2001b).

Due to its small velocity dispersion the Fornax cluster has so far been assumed to have no substructure. However, Drinkwater et al. (2001a) have analysed the dynamics of the cluster and find definite evidence for a subcluster in the South West of the cluster, centred on Fornax A (NGC 1316). In fact, a slightly enhanced galaxy density in this region can also be seen in Fig. 16 of the FCC. If we exclude the galaxies in this subcluster from the TullyFisher relation we obtain

$B_{\mathrm{T}}^{\mathrm{o}, i}(\mathrm{~F})=-6.50 \cdot\left[\log V_{\mathrm{M}}-2.0\right]+(12.69 \pm 0.12)$,

as shown in Fig. 7, with a scatter of 0.50. The scatter is considerably smaller than before and comparable to the intrinsic scatter, which is about 0.4 (cf. Sakai et al. 2000 find 0.43 for near-by galaxies with Cepheid distances).

We have not attempted to derive a Tully-Fisher relation of the subcluster alone since only six galaxies therein are useful for the Tully-Fisher relation. Furthermore, one of these has unusual $\mathrm{H}$ I masses due to interactions (NGC 1316C, cf. Sect. 4). However, in the mean they are $(1.0 \pm 0.5)$ mag brighter than the main cluster, indicating that the subcluster lies in the foreground of Fornax (which is also supported by the lower Cepheid distance of NGC 1326A, 18.7 Mpc, in the subcluster). In combination with its slightly larger mean velocity of $1583 \mathrm{~km} \mathrm{~s}^{-1}$ (as compared to $1478 \mathrm{~km} \mathrm{~s}^{-1}$ for the main cluster, Drinkwater et al. 2001a) we now have a three-dimensional picture of the subcluster in the foreground falling into the main cluster. The high rate of $\mathrm{H}$ I detection in the subcluster (see Fig. 2) suggests that it is falling into the main cluster for the first time. This is consistent with the slightly lower H I deficiency $(0.2 \pm 0.2)$ we measure for the subcluster galaxies compared to the rest of the cluster $(0.44 \pm 0.10)$.

The Tully-Fisher relation for the main cluster can be used to derive a relative distance to the Virgo cluster as shown by Schröder (1995). Using the same parameters and corrections where possible (see description above; morphological types and axial ratios were taken from the $\mathrm{VCC}^{5}$, H I line widths from Bottinelli et al. 1990) we find

$B_{\mathrm{T}}^{\mathrm{o}, i}(\mathrm{~V})=-(6.50 \pm 0.36) \cdot\left[\log V_{\mathrm{M}}-2.0\right]+(13.07 \pm 0.07)$

for Virgo, with a scatter of $0.64 \mathrm{mag}$. This relation includes all galaxies classified as members of the Virgo cluster proper (Binggeli et al. 1993). We find a relative distance of $-0.38 \pm 0.14 \mathrm{mag}$ with the Fornax cluster being closer.

\footnotetext{
5 Virgo Cluster Catalog (Binggeli et al. 1985).
}

This is consistent with other Tully-Fisher distances between Fornax and Virgo (Bureau et al. 1996: $-0.06 \pm 0.15$; Aaronson et al. 1989: $-0.25 \pm 0.23$; Visvanathan 1983: $-0.20 \pm 0.18)$, contrary to some other distance measurements that place Fornax further away (e.g., McMillan et al. 1993: $0.24 \pm 0.10$, using the planetary nebulae luminosity function). The relative distance measurements found in the literature vary from $-0.5 \mathrm{mag}$ to $+0.4 \mathrm{mag}$ (see Table 6.1 in Schröder 1995; Table 3 in Bureau et al. 1996). However, many methods use only a small sample of galaxies (e.g., type Ia supernovae, surface brightness fluctuations, planetary nebulae luminosity functions) where the cluster centres are less well defined.

The Tully-Fisher relation of the spatially more confined Virgo subcluster $B$ with 20 galaxies shows a smaller scatter of 0.46 , similar to the one of the Fornax cluster galaxies and of the calibrators. This agrees well with the velocity dispersion of the $B$ cluster being significantly smaller than that of the whole Virgo cluster ( $499 \mathrm{~km} \mathrm{~s}^{-1}$ vs. $699 \mathrm{~km} \mathrm{~s}^{-1}$, see Binggeli et al. 1993). We find that the local intercept is fainter $(13.21 \pm 0.11)$ and the relative distance larger: $-0.52 \pm 0.16$ mag.

\section{Conclusion}

In this paper we have presented deep H I observations of all spiral galaxies as well as bright early-type galaxies in the FCC. Two late-type spiral galaxies and three irregulars were not detected, possibly because of an interference at $v \simeq 1250 \mathrm{~km} \mathrm{~s}^{-1}$. Only one out of four Sa galaxies was detected, and none of the S0/a galaxies and galaxies of earlier morphological types.

The distribution of galaxies with $\mathrm{H}$ I in the Fornax cluster differs significantly from the more centrally concentrated distribution of non-H I detections, as expected from the density-morphology relation. Even the $\mathrm{H}$ I-rich galaxies are more centrally concentrated around the cluster than a random distribution: this shows that they are aware of the cluster potential. However, there is no significant difference in the velocity distribution of the two samples.

The mean $M_{\mathrm{HI}} / L_{B}$ binned by morphological type of the Fornax galaxies is between those of the Virgo galaxies and of galaxies in the field indicating a modest but significant $\mathrm{HI}$ depletion: the mean $M_{\mathrm{HI}} / L_{B}$ is $60 \pm 13 \%$ the value for a comparison sample of field galaxies. In addition, the $\mathrm{HI}$ deficiency parameter, as introduced by Solanes et al. (1996), is $0.38 \pm 0.09$, which is significantly greater than zero. There is some indication from the kinematics of the galaxies that this H I depletion is caused by ram-pressure stripping of galaxies which are on orbits that pass closer to the cluster core. In addition, optical observations show evidence of enhanced star formation in galaxies in these outer parts of the cluster which will use up some of the gas.

We have calculated the $B$-band Tully-Fisher relation relation for the Fornax cluster from our data and obtain a good fit for the main cluster with a scatter of $0.50 \mathrm{mag}$. The relative distance to the Virgo cluster is 

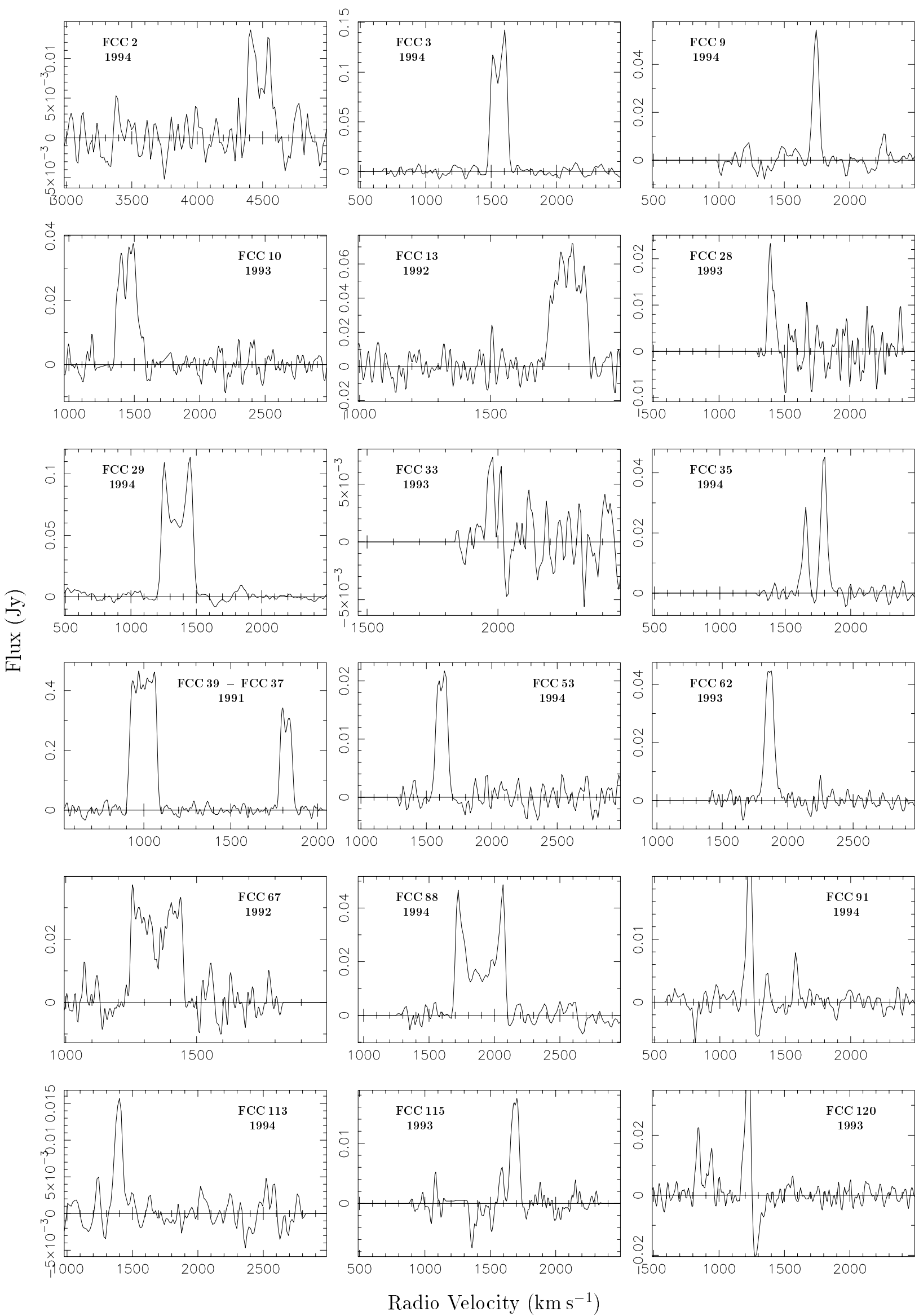

Fig. 8. H I spectra of the detected galaxies in the Fornax cluster. In each plot the flux in Jy is plotted versus the velocity in $\mathrm{kms}^{-1}$. 

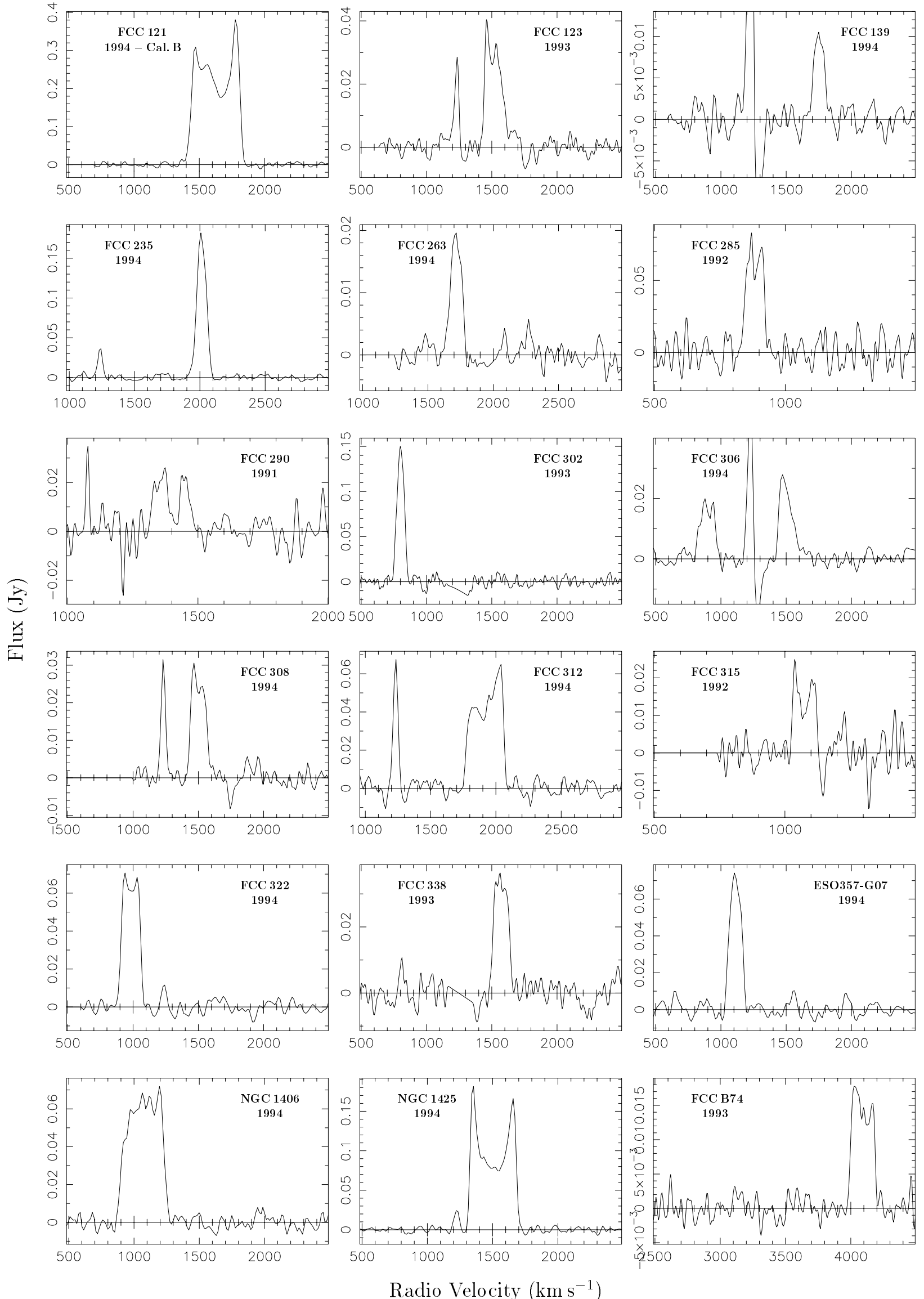

Fig. 8. continued. 
$-0.38 \pm 0.14 \mathrm{mag}$ with the Fornax cluster being closer. The Tully-Fisher relation confirms the existence of a subcluster in the South-West of the main cluster, centred on NGC 1316 (Fornax A). The subcluster galaxies are almost one magnitude brighter than the main cluster. Combined with their higher mean velocity and the higher $\mathrm{HI}$ detection rate, this indicates that the subcluster lies in the foreground and is currently falling into the cluster for the first time.

Acknowledgements. We thank the staff at the Parkes Observatory, in particular Euan Troop, for making our stays at the telescope very pleasant and productive. Several people helped us in conducting the observations at the Parkes telescope, notably M. G. McMaster (STScI), R. Otrupcek (ATNF), R. C. Kraan-Korteweg (Univ. of Guanajuato), and P. Henning (Univ. of New Mexico). We are very grateful to M. Waugh and M. Marquarding for making some HIPASS measurements available prior to publication, and to M. Zwaan for helpful discussions. We wish to thank the referee of this paper for detailed comments which greatly improved the presentation of this work. A. Schröder gratefully acknowledges financial support from the European Marie Curie Fellowship Grant.

\section{Appendix A: H I spectra}

The H I spectra (uncalibrated) for all galaxies detected during our Parkes observations are shown in Fig. 8. The calibration factor varied from year to year: 1.00 for 1991 , 1.25 for 1992, 1.28 for 1993, and 1.32 for 1994 . We show one spectrum per galaxy (because of the different set-ups per year we did not average the spectra of the different years), the others are available from one of the authors (Schröder).

During the 1993 and 1994 observing runs, data suffered frequently from strong interference at a central radial velocity of $1250 \mathrm{~km} \mathrm{~s}^{-1}$. Negative spikes were excluded by range selection but positive spikes are sometimes easily visible in the plotted spectra.

\section{References}

Aaronson, M., Bothun, G. D., et al. 1989, ApJ, 338, 654

Abadi, M. G., Moore, B., \& Bower, R. G. 1999, MNRAS, 308, 947

Abell, G. O. 1958, ApJS, 3, 211

Barnes, D. G., Staveley-Smith, L., Webster, R. L., \& Walsh, W. 1997, MNRAS, 288, 307

Binggeli, B., Sandage, A., \& Tammann, G. A. 1985, AJ, 90, 1681 (VCC)

Binggeli, B., Popescu, C., \& Tammann, G. A. 1993, A\&AS, 98, 275

Bothun, G. D., Schommer, R. A., \& Sullivan, W. T. 1984, AJ, 89,466

Bottinelli, L., Gouguenheim, L., Fouqué, P., \& Paturel, G. 1990, A\&AS, 82, 391

Bravo-Alfaro, H., Cayatte, V., van Gorkom, J. H., \& Balkowski, C. 2000, AJ, 119, 580
Bridges, T. J., Hanes, D. A., \& Harris, W. E. 1991, AJ, 101, 469

Bureau, M., Mould, J. R., \& Staveley-Smith, L. 1996, ApJ, 463,60

Cayatte, V., Kotanyi, C., Balkowski, C., \& van Gorkom, J. H. 1994, AJ, 107, 1003

da Costa, L. N., Pellegrini, P. S., Davis, M., et al. 1991, ApJS, 75,935

de Vaucouleurs, G., de Vaucouleurs, A., Corwin, H. G., et al. 1991, Third Reference Catalogue of Bright Galaxies, vols. I - III (Springer, New York) (RC3)

Drinkwater, M. J., Gregg, M., D., \& Colless, M. 2001a, ApJ, 548, L139

Drinkwater M. J., Gregg M. D., Holman, B., \& Brown, M. 2001b, MNRAS, in press

Ferguson, H. C. 1989a, AJ, 98, 367 (FCC)

Ferguson, H. C. 1989b, Ap\&SS, 157, 227

Ferguson, H. C., \& Binggeli, B. 1994, A\&AR, 6, 67

Freedman, W. L., et al. 2001, ApJ, 553, 47

Fouqué, P., Bottinelli, L., Durand, N., Gouguenheim, L., \& Paturel, G. 1990, A\&AS, 86, 473

Giovanelli, R., \& Haynes, M. P. 1985, ApJ, 292, 404

Guhathakurta, P., van Gorkom, J. H., Kotanyi, C. G., \& Balkowski, C. 1988, AJ, 96851

Hamuy, M., Phillips, M. M., Maza, J., et al. 1991, AJ, 102, 208

Haynes, M. P., \& Giovanelli, R. 1984, AJ, 89, 758

Heidmann, J., Heidmann, N., \& de Vaucouleurs, G. 1971, Mem. R. Astron. Soc., 75, 85

Helou, G., Giovanardi, C., Salpeter, E. E., \& Krumm, N. 1981, ApJS, 46, 267

Helou, G., Hoffman, G. L., \& Salpeter, E. E. 1984, ApJS, 55, 433

Hoffman, G. L., Helou, G., Salpeter, E. E., Glosson, J., \& Sandage, A. 1987, ApJS, 63, 247

Hoffman, G. L., Helou, G., \& Salpeter, E. E. 1988, ApJ, 324, 75

Hoffman, G. L., Lewis, B. M., Helou, G., Salpeter, E. E., \& Williams, H. L. 1989, ApJS, 69, 65

Horellou, C., Casoli, F., \& Dupraz, C. 1995, A\&A, 303, 361

Huchtmeier, W. K., \& Richter, O.-G. 1988, A\&A, 203, 237

Huchtmeier, W. K., \& Richter, O.-G. 1989a, A\&A, 210, 1

Huchtmeier, W. K., \& Richter, O.-G. 1989b, A General Catalog of H I Observations of Galaxies (Springer Verlag, New York) (HR89)

Jones, C., Stern, C., Forman, W., et al. 1997, ApJ, 482, 143

Killeen, N. E. B., \& Bicknell, G. V. 1988, ApJ, 325, 165

Kolatt, T., Dekel, A., \& Lahav, O. 1995, MNRAS, 275, 797

Lauberts, A., \& Valentijn, E. A. 1989, The Surface Photometry Catalog of the ESO-Uppsala galaxies, European Southern Observatory, Garching bei München

Loveday, J. 1996, MNRAS, 278, 1025

MacGillivray, H. T., Beard, S. M., \& Dodd, R. J. 1988, Alds. Proc. 389

Marquarding, M. 2000, M.Sc. Thesis, Univ. of Melbourne

Madore, B. F., et al. 1999, ApJ, 515, 29

Mathewson, D. S., Ford, V. L., \& Buchhorn, M. 1992, ApJS, 81,413

McMahon, P., van Gorkom, J. H., Richter, O.-G., \& Ferguson, H. C. 1992, AJ, 103, 399

McMillan, R., Ciardullo, R., \& Jacoby, G. H. 1993, ApJ, 416, 62

Mould, J. R., et al. 2000, ApJ, 528, 655

Nilson, P. 1973, Uppsala General Catalog of Galaxies, Astr. Obs. Publ., Uppsala (UGC) 
Paturel, G., Fang, Y., Garnier, R., Petit, C., \& Rousseau, J. 2000, A\&AS, 146, 19

Pierce, M. J. 1989, ApJ, 344, L57

Prosser, C. F., et al. 1999, ApJ, 525, 80

Putman, M., Bureau, M., Mould, J. R., Staveley-Smith, L., \& Freeman, K. C. 1998, AJ, 115, 2345

Richter, O.-G., \& Huchtmeier, W. K. 1984, A\&A, 132, 253

Richter, O.-G., \& Sadler, E. M. 1985, A\&AS, 59, 433

Sakai, S., Mould, J. R., et al. 2000, ApJ, 529, 698

Sandage, A., \& Tammann, G. A. 1981, 1987, A Revised Shapley-Ames Catalog of Bright Galaxies, Carnegie Institution Publ. 635, Washington (RSA)

Schröder, A. 1995, Ph.D. Thesis, Univ. of Basel

Schröder, A., \& Visvanathan, N. 1996, A\&AS, 118, 441
Solanes, J. M., Giovanelli, R., \& Haynes, M. H. 1996, ApJ, 461, 609 (SGH)

Solanes, J. M., Manrique, A., García-Gómez, C., et al. 2001, ApJ, 548, 97

Staveley-Smith, L., et al. 1996, PASA, 13, 243

van Gorkom, J. H. 1996, in The Minnesota Lectures on Extragalactic Neutral Hydrogen, ed. E. D. Skillman

Visvanathan, N. 1983, ApJ, 275, 430

Waugh M., Webster R. L., \& Drinkwater, M. J. 2000, in Mapping the Hidden Universe, ed. R. C. Kraan-Korteweg, P. A. Henning, \& H. Andernach, ASP Conf. Ser., 218, 231

Wright, A. E., \& Otrupcek R. 1990, Parkes Catalogue, Monograph Australia Telescope National Facility (Publisher) 